\title{
CARACTERIZACIÓN FÍSICO-QUÍMICA DE HARINA DE TRIGO, MASA Y PAN
}

\section{PHYSICAL-CHEMICAL CHARACTERIZATION OF WHEAT FLOUR, DOUGH AND BREAD}

\author{
Jairo Montoya López y Germán Antonio Giraldo Giraldo². \\ ${ }^{1}$ Estudiante Maestría en Química, énfasis alimentos. Universidad del Quindío. \\ ${ }^{2}$ Director Grupo de Investigación de Agroindustria de Frutas Tropicales, Laboratorio de diseño de nuevos productos. Universidad del Quindío.
}

Fecha de recibido: Febrero 3 de 2010

Fecha de aceptado: Junio 9 de 2010

Correspondencia: Laboratorio Diseño de Nuevos Productos, Programa de Maestría en Química, Universidad del Quindío, Av. Bolívar calle 12 norte Armenia Quindío. Correo electrónico: jmontoya@uniquindio.edu.co

\section{RESUMEN}

El objetivo de este trabajo es evaluar algunos componentes químicos en una harina de trigo comercial, la masa y el pan elaborado. A la harina se le determinó el índice de blancura; a la harina de trigo comercial y al pan se les realizo análisis de calorimetría diferencial de barrido (DSC) y análisis termogravimétrico (TGA); a la harina, la masa y el pan se les realizo análisis de humedad, actividad de agua (aw) y determinación de color. Los resultados mostraron un cambio de color significativo de la masa $(\Delta E=5.4191)$ y del pan $(\Delta E=12.7314)$ respecto a la materia prima $(\Delta E=0)$, los resultados de la humedad y de la actividad de agua, fueron los esperados comparados con los datos encontrados en la bibliografía. Otros componentes evaluados fueron: Determinación de DSC y TGA a la harina de trigo lo que permitió la identificación de estructuras que son consecuencia de las condiciones de procesamiento como las generadas durante la transición vítrea, la gelatinización y la retrogradación del almidón, al igual que las modificaciones térmicas por los tratamientos combinados de calor y humedad, los cuales cambian las propiedades fisicoquímicas del almidón sin destruir su estructura granular, igualmente en la determinación de DSC y TGA para el pan, la transición vítrea se induce por el cambio de temperatura de un polímero amorfo vítreo a un estado progresivamente gomoso cuando este se calienta.

Palabras clave: Fortificación, Análisis físico-Químico, Harina de Trigo, Alimentos

\begin{abstract}
The aim of this study was to evaluate some chemical components in a commercial wheat flour, dough and bread made. Whiteness index was determined on the wheat. Analysis of differential scanning calorimetry (DSC) and thermogravimetric analysis (TGA) test were conducted on bread and commercial wheat flour. To flour, bread and dough humidity tests, water activity (aw) and color determination were conducted. The results showed a significant color change of the dough $(\triangle E=$ 5.4191) and bread $(\Delta E=12.7314)$ for the raw material $(\Delta E=0)$, the results for humidity and water activity, were as expected compared with data found in literature. Other components evaluated were: determination of DSC and TGA to wheat flour which allowed the identification of structures that result from processing conditions like those generated during the glass transition, gelatinization and retrogradation of starch, as the thermal modifications by the combined treatments of heat and humidity, which change the physicochemical properties of starch without destroying its granular structure, also in the determination of DSC and TGA for bread, the glass transition is induced by the temperature change of an amorphous glassy polymer to a gradually rubbery state when it is heated
\end{abstract}

Keywords: Fortification, Physical-Chemical analysis, Wheat Flour, Food

\section{1. INTRODUCCIÓN}

La fortificación de alimentos de consumo habitual y la suplementación de nutrientes deficitarios, es una estrategia promovida por el Estado[1], con el fin de prevenir de algún modo la malnutrición o la falta de micronutrientes, la desnutrición por micronutrientes es un problema extendido mundialmente que tiene tantas consecuencias sobre la salud como económicas, la fortificación de alimentos de consumo habitual y la suplementación de nutrientes deficitarios en la alimentación buscan prevenir la malnutrición por deficiencia de micronutrientes,[2] la fortificación es una de las estrategias más importantes para aumentar la ingesta de las vitaminas y los minerales de importancia para la salud pública y mejorar el estado de nutrición de las personas, de forma continua y auto sostenida.[3] siendo la harina de trigo el vehículo más utilizado para esta fortificación, esta harina es utilizada como materia prima para la elaboración de productos de panadería, pastas, sopas, coladas, entre otros [4]; los cuales requieren temperaturas superiores a 100 o C, lo que posiblemente cause transformación en los componentes nutricionales de la materia prima, entre ellos la degradación de las vitaminas por su carácter termolábil e hidrosoluble, igualmente el refinado elimina muchas vitaminas, muchas fibras y buena parte de los minerales; por ejemplo, la harina integral refinada pierde el $100 \%$ de

Rev. Invest. Univ. Quindío (20): 29-35. Armenia - Colombia 
vitamina $A$; también la cocción destruye una buena parte de vitaminas, bioflavonoides y altera la estructura proteica. Las vitaminas termolábiles como la Co las fotolábiles como la B6 se destruyen completamente con la cocción, sucede lo mismo con muchos otros bioflavonoides, sensibles a la variación de temperatura[5]. La vitamina B2 es estable ante el calor y al contacto con el aire (oxidación). Sin embargo, es sensible a la luz. La luz es un factor primario para su destrucción. La vitamina B1 o tiamina es altamente inestable, por ello se pierde ante la cocción[5], según la temperatura y la acidez del medio $(\mathrm{pH})$. La congelación por un periodo prolongado de alimentos con contenido de vitamina B1 puede resultar en una pérdida significativa de la misma (entre 20 y $60 \%$ )

Teniendo como premisa que la desnutrición es multicausal, el Estado ha diseñado una serie de programas de intervención orientados a la prevención y al control de la desnutrición[6]. El gobierno de Colombia, bajo la iniciativa del Ministerio de Salud Pública, promovió el decreto 1944 de 1996. [3] que aprueba una fortificación obligatoria de la harina de trigo con vitamina B1, vitamina B2, niacina, acido fólico y hierro. Logrando que la fortificación se implementara en un $100 \%$. Las deficiencias de vitaminas y minerales afectan especialmente a madres y niños en áreas rurales y marginales de las grandes ciudades de los países en desarrollo[7], debido al consumo insuficiente de alimentos ricos en estos nutrientes, a infecciones recurrentes y síndromes de mala absorción. [2] Sin embargo, en mérito a la deficiencia de micronutrientes causa más de la mitad de los casos de desnutrición en todo el mundo, los programas de suplementación y fortificación de alimentos, constituyen las mejores intervenciones para la lucha contra la desnutrición[6].

Las investigaciones realizadas hasta la fecha, se han fundamentado especialmente en el seguimiento de las poblaciones que se han sometido al enriquecimiento[4] de la harina, dando muy buenos resultados, sin embargo dentro de este proceso hace falta la comprobación y el sustento por medio de análisis químicos de seguimiento, por la crucial importancia que siempre ha tenido y tendrá al análisis de alimentos, el cual constituye una de las herramientas indispensables para el estudio y aplicación del control de calidad y para llevar a cabo cualquier desarrollo que implique medir características como en este caso con la harina de trigo.

La mayoría de los ensayos para medir la eficacia de la fortificación se realiza mediante un seguimiento a los individuos[8] y no mediante el seguimiento físico-químico de las concentraciones de los micronutrientes tanto para la materia prima, como para los productos terminados, algunos autores reportan análisis de las concentraciones de hierro, calcio y zinc en el sector de los cereales y legumbres[4] habitualmente consumidos por los niños que viven en Indonesia utilizando espectrofotometría de llama de absorción atómica, debido a que hay escases de análisis que permitan tener datos verdaderos sobre el contenido de minerales en alimentos complementarios para Asia, el análisis se centra de este modo en los cereales y leguminosas consumidos por los lactantes indonesios determinándose las concentraciones de hierro, zinc y calcio utilizando el método espectrofotometría de llama de absorción atómica y la cromatografía líquida de alta resolución (HPLC)[9]. El trabajo pretende implementar herramientas de optimización en la determinación de la eficacia del proceso de fortificación de la harina de trigo y de los productos elaborados a base de ella, los análisis más desarrollados y más reportados en la literatura tienen que ver con los de la fijación del hierro y del ácido fólico. No obstante las nuevas investigaciones se pueden direccionar a la comprobación de la efectividad de la fortificación de las harinas mediante análisis físico-químico de la harina fresca y los productos alimenticios que la utilizan como materia prima.

Este trabajo es un estudio preliminar desarrollado con el objetivo de evaluar algunos componentes físicos presentes en la harina de trigo y un producto elaborado como lo es el pan con análisis como TGA, DSC y fisicoquímicos de la harina de trigo, la masa y el pan, determinando parámetros como: humedad, color y actividad de agua.

\section{MATERIALES Y MÉTODOS}

\subsection{Materia Prima}

Se utilizo una muestra de harina de trigo a la cual se le aplico la técnica del cuarteo, que consiste en recoger el material de diferentes puntos del empaque, en una cantidad superior a la necesaria para el ensayo. Este material se distribuye en cuatro cuadrantes, previa homogenización, y se recoge el correspondiente a dos cuadrantes opuestos, que se vuelve a mezclar y a presentar como cuatro cuadrantes, procediéndose de la misma manera, hasta llegar a conseguir la cantidad de muestra necesaria; la selección de la muestra de la masa se hace seleccionando un pedazo de la masa original, la cual se prepara siempre a las mismas condiciones, igualmente el pan se selecciona una muestra al azar de la producción total.

\subsection{Materiales y Métodos}

Una vez adecuadas las materias primas se colocaron las muestras en la estufa a $105^{\circ} \mathrm{C}$; durante 48 horas por triplicado en una estufa de recirculación forzada marca Blinder (norma NTC 282). La determinación de color se realizo en un medidor de color marca minolta, determinando los valores $\mathrm{L}, \mathrm{a}, \mathrm{b}, \mathrm{c}$ y $\mathrm{h}$ y la determinación de actividad de agua utilizando un equipo de medición del contenido de agua por punto de roció marca aqualab. El pan se secó en una estufa marca Blinder a $105^{\circ} \mathrm{C}$ durante 12 horas, fue rayado empleando un rayador de acero inoxidable y pasado por un tamiz de $600 \mathrm{~mm}$ obteniéndose la harina de pan. La determinación de la temperatura y la entalpia de Gelatinización ( $\mathrm{Tp}$ y Hp) de cada una de las muestras se llevo a cabo por medio de un Calorímetro Diferencial de Barrido (TA Instruments DSC-Q100), en muestras de (10 \pm 0.5$) \mathrm{mg}$ con el contenido de humedad respectivo cada muestra, genero una velocidad de calentamiento de $5{ }^{\circ} \mathrm{C} / \mathrm{min}$, desde 
temperatura ambiente hasta $100^{\circ} \mathrm{C}$, en atmosfera de Nitrógeno. La descomposición térmica de las muestras fue estudiada a través de la técnica de análisis termogravimétrico (TA Instruments TGA Q500), realizando experimentos en presencia de una atmosfera de Nitrógeno para un rango temperaturas desde ambiente hasta $800^{\circ} \mathrm{C}$ a $5^{\circ} \mathrm{C} / \mathrm{min}$ y en muestras de $(6 \pm 0.5) \mathrm{mg}$.

La determinación del índice de blancura de la harina comercial se realizó con un colorímetro HunterLab Color Quest XE calibrado con un estándar blanco en forma de placa ( $L=97.79, a=-0.53$ y $b=+2.28$ ), leyendo con un iluminante de tipo $\mathrm{C}$, obteniendo los factores $\mathrm{a}$, b y L del sistema Hunter.

\section{RESULTADOSY DISCUSIÓN}

\section{Calorimetría Diferencial de Barrido}

La aplicación de DSC que se presenta en la Figura 1 ha permitido la identificación de estructuras que son consecuencia de las condiciones de procesamiento como las generadas durante la transición vítrea, la gelatinización y la retrogradación del almidón, al igual que las modificaciones térmicas por los tratamientos combinados de calor $y$ humedad, los cuales cambian las propiedades fisicoquímicas del almidón sin destruir su estructura granular.

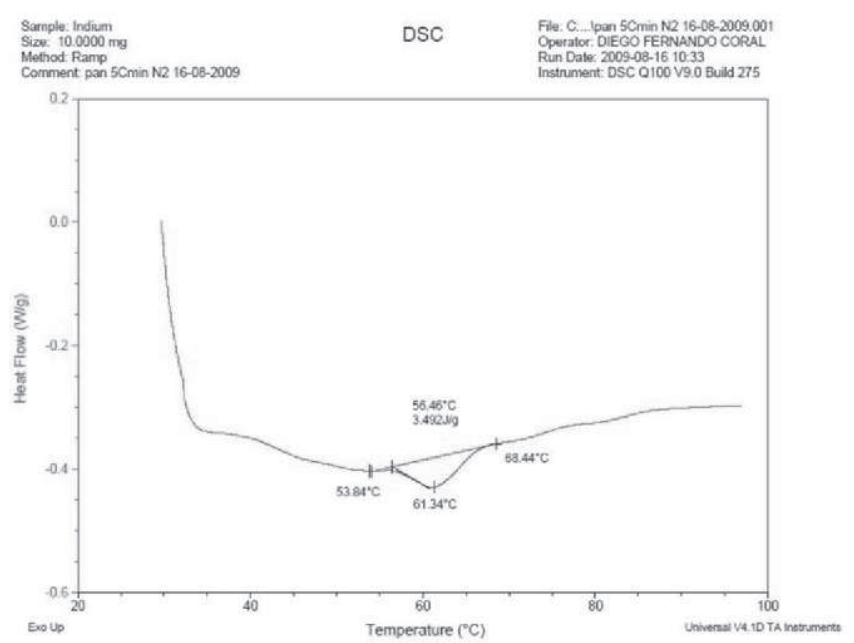

Figura 1. Termograma DSC de la Harina de trigo

El termograma DSC de la harina presenta la transición térmica de gelatinización. La gelatinización se presenta como un suave pico endotérmico sobre la línea base del termograma. El proceso, aquí representado, empieza a 53.27 oC, esta temperatura se conoce como temperatura inicial (To), la temperatura de pico (Tp) es la temperatura donde se registran los valores más altos de absorción de calor, para la harina esta temperatura es de $61.96 \stackrel{\circ}{\circ}$, a medida que el proceso finaliza, el sistema regresa a un estado en el cual no hay cambios en la fase ni en la composición de la muestra. La energía necesaria para completar el proceso se conoce como entalpía de gelatinización (Hp) y para la harina su valor es de $1.080 \mathrm{~J} / \mathrm{g}$.

A medida que la temperatura aumenta, la muestra absorbe calor del medio para producir la fusión de los cristales de amilopectina y la hidratación de las moléculas de amilosa tal como se ve en el proceso de gelatinización y también al inicio del proceso donde se presenta un proceso endotérmico, lo cual se explicaría por la absorción de calor que se requiere al principio del proceso. La medida se realiza comparando la absorción o emisión de calor de la charola de referencia (línea base) con la absorción o emisión de calor de la charola en donde se encuentra la muestra, si ningún proceso termodinámico ocurre los dos termogramas son iguales, pero si existen modificación de algún tipo en la muestra existirá una diferencia en los termogramas, esta diferencia puede interpretarse como un proceso endotérmico (diferencia negativa) o exotérmico (diferencia positiva). El área que se encierra entre la línea base y la línea de la muestra brinda información sobre la entalpía del proceso. La entalpía es la energía necesaria para llevar a cabo la transición termodinámica.

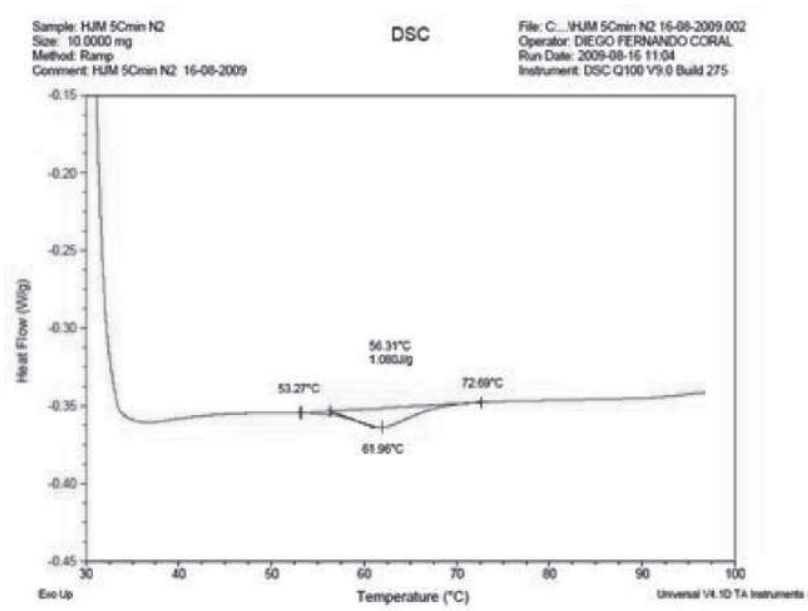

Figura 2. Termograma DSC del Pan

La transición vítrea se induce por el cambio de temperatura de un polímero amorfo vítreo a un estado progresivamente gomoso cuando se calienta [10]. En DSC, la evidencia directa de la transición vítrea se caracteriza por un incremento en la capacidad calorífica $(\Delta \mathrm{Cp})$ de la muestra, la cual al ser reversible puede ser medida durante el calentamiento o enfriamiento [11]. Sin embargo, la determinación con exactitud de esta temperatura es difícil principalmente porque el cambio es pequeño y muchas veces se encuentra sumergido en la línea base [10].

En el termograma del PAN se detectaron cambios en el calor específico $(\triangle C p)$ aproximadamente en un rango de temperatura entre 30 y $40^{\circ} \mathrm{C}$, rango en el cual posiblemente se encuentre la temperatura de transición vítrea.

En este termograma también se presenta el proceso de gelatinización con las siguientes temperaturas

$\mathrm{To}=53.84^{\circ} \mathrm{C}$

$\mathrm{Tp}=61.34^{\circ} \mathrm{C}$

Rev. Invest. Univ. Quindío (20): 29-35. Armenia - Colombia 
$\mathrm{Tf}=68.44^{\circ} \mathrm{C}$

$\mathrm{Hp}=3.492 \mathrm{~J} / \mathrm{g}$

La temperatura pico o temperatura de gelatinización es donde se pierde la estructura cristalina de los gránulos de almidón, por la tanto una temperatura más alta indica un arreglo cristalino más estable.

El hinchamiento de los gránulos de almidón[12] que se presenta durante la gelatinización provoca que la viscosidad del medio se incremente. Este proceso es endotérmico y se requiere de aproximadamente de $10 \mathrm{~J} / \mathrm{g}$ de almidón para llevarlo acabo, como lo han demostrado estudios de calorimetría diferencial de barrido DSC [11].

El intervalo de temperatura de la transición, es decir la diferencia entre la temperatura final y la temperatura de inicio es un indicador del nivel de heterogeneidad de los cristales, al ser mayor el intervalo, mayor heterogeneidad de los cristales presentes en la muestra.

\section{Análisis Termogravimétrico}

El Termograma TGA (línea verde) y su derivada (DTG) (línea azul), para la harina es mostrado en la figura 3 en el cual se registraron las pérdidas de peso de la muestra al ser sometidas a altas temperaturas. Con los resultados obtenidos se determinaron las pérdidas o ganancias de peso debido a descomposición, oxidación y deshidratación.

La Figura 3 se ha dividido en tres regiones: 1, 2 y 3, relacionadas con las pérdidas de masa más pronunciadas en la gráfica.

La zona uno corresponde a la pérdida de masa corresponde a la humedad presente en la muestra donde la cantidad de agua disponible fue de $12,42 \%$, esta disminución se presento entre los 100 y $200{ }^{\circ} \mathrm{C}$ presentando su mayor pico alrededor de $\operatorname{los} 150^{\circ} \mathrm{C}$

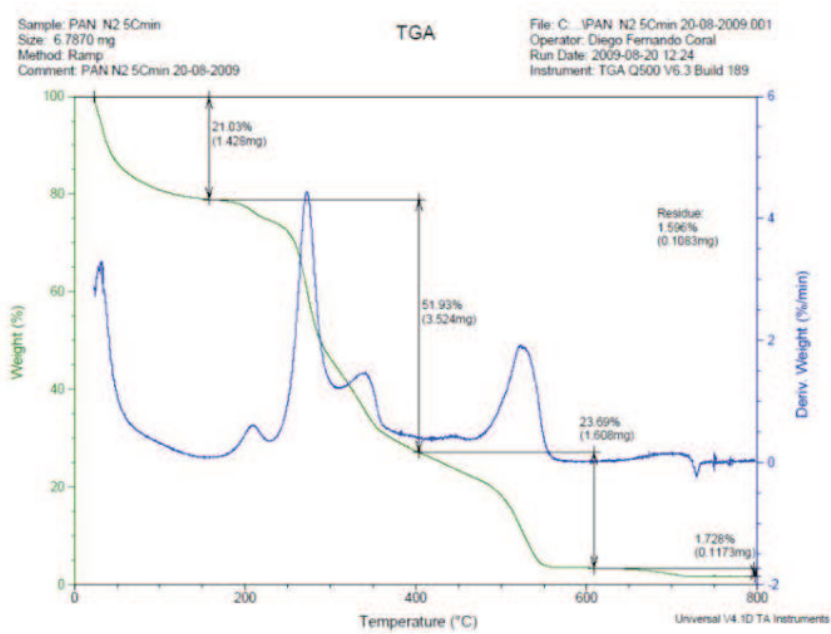

Figura 3. TGA y derivada DTG de Harina de Trigo
La pérdida de peso más representativa se presento en la zona 2 que se presenta en rango de temperaturas entre $200^{\circ} \mathrm{C}$ y $400^{\circ} \mathrm{C}$ teniendo la velocidad máxima de descomposición aproximadamente en $300^{\circ} \mathrm{C}$, en este punto se descomponen carbohidratos, péptidos de bajo peso molecular y la cantidad total de almidón presente en la muestra ya que esta pérdida de peso se encuentra en el rango de temperaturas donde se da la degradación del almidón. En esta fase la pérdida de peso fue de $63,49 \%$. Para este estudio es de gran importancia esta zona que representa la mayor pérdida de masa de la muestra y está en el rango de descomposición del almidón. La descomposición del almidón debida a la temperatura se presenta como una curva característica de una descomposición simple o de un solo paso como las presentadas en la (Figura 3) esto se debe a que la muestra contiene grandes cantidades de carbohidratos totales los cuales se descomponen a las temperaturas comprendidas en la zona 2.

En la zona 3 con un rango de temperaturas entre $400^{\circ} \mathrm{C}$ a $600^{\circ} \mathrm{C}$ con un pico máximo presentado aproximadamente en $500^{\circ} \mathrm{C}$ se descomponen polisacáridos de alto peso molecular como proteínas, lípidos entre otros compuestos orgánicos. En este punto la muestra presento una pérdida de peso de $23,54 \%$.

El porcentaje de residuos presentados en el termograma (Figura 3) son del 0,4227\%. Este valor es mínimo lo que indica que la muestra contiene bajas concentraciones de minerales y lo que pueden ser al igual sales acopladas.

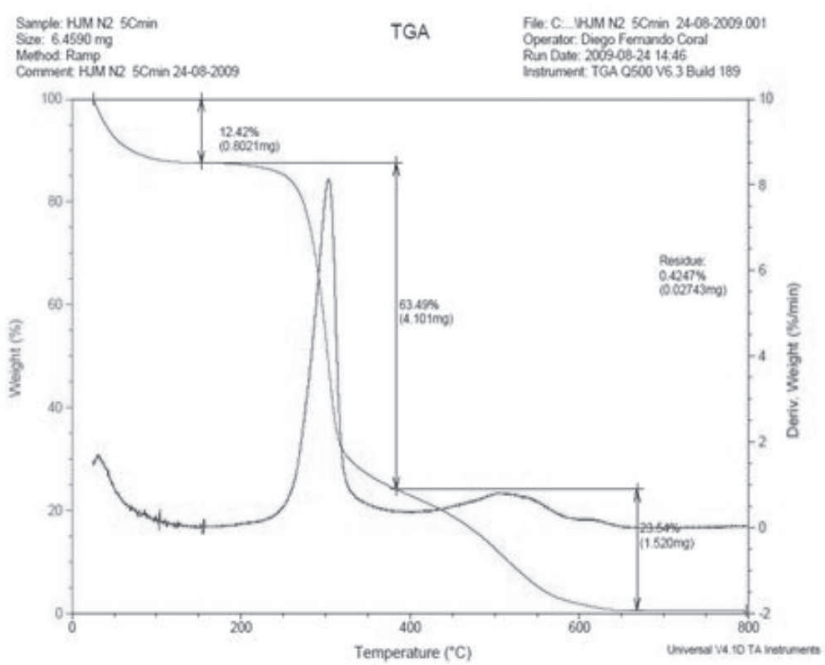

Figura 4. TGA y derivada DTG de Pan

La Figura 4 se ha dividido en tres regiones: 1, 2 y 3, relacionadas con las pérdidas de masa más pronunciadas en la gráfica. La zona uno corresponde a la pérdida de masa corresponde a la humedad presente en la muestra donde la cantidad de agua disponible fue de $21,03 \%$, esta disminución se presento entre los 100 y $200^{\circ} \mathrm{C}$ presentando su mayor pico alrededor de $\operatorname{los} 150^{\circ} \mathrm{C}$. 
La pérdida de peso más representativa se presento en la zona 2 que se presenta en rango de temperaturas entre $200^{\circ} \mathrm{C}$ y $400^{\circ} \mathrm{C}$ teniendo la velocidad máxima de descomposición aproximadamente en $250^{\circ} \mathrm{C}$, en este punto se descomponen carbohidratos, péptidos de bajo peso molecular y la cantidad total de almidón presente en la muestra ya que esta pérdida de peso se encuentra en el rango de temperaturas donde se da la degradación del almidón. En esta fase la pérdida de peso fue de 51,93\%. La descomposición del almidón debida a la temperatura se presenta como una curva característica de una descomposición simple o de un solo paso como las presentadas en la (figura 4) esto se debe a que la muestra contiene grandes cantidades de carbohidratos totales los cuales se descomponen a las temperaturas comprendidas en la zona 2.

En la zona 3 con un rango de temperaturas entre $400^{\circ} \mathrm{C}$ a $550^{\circ} \mathrm{C}$ con un pico máximo presentado aproximadamente en $530^{\circ} \mathrm{C}$ se descomponen polisacáridos de alto peso molecular como proteínas, lípidos entre otros compuestos orgánicos. En este punto la muestra presento una pérdida de peso de $23,69 \%$. El porcentaje de residuos presentados en el termograma Figura 6 es del 1,596\%.

\section{Índice de Blancura}

La medición del índice de blancura utilizando el colorímetro HunterLab arrojó los siguientes resultados:

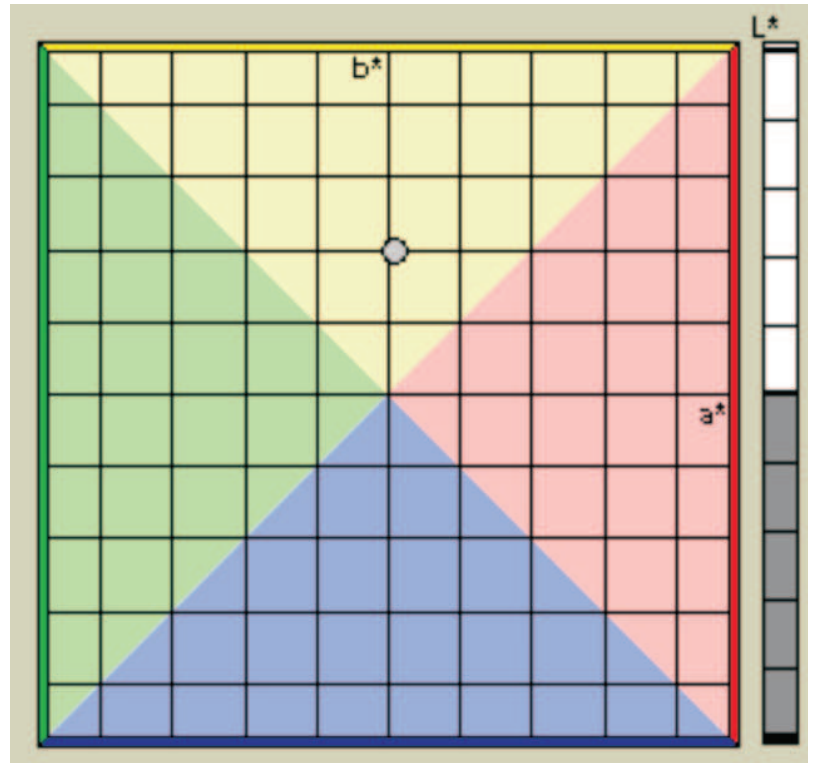

Figura 5. Factores $L^{*}, a^{*} y b^{*}$ del sistema Hunter.

El parámetro L presentó un valor alto de 92.01 unidades: las muestras tuvieron una luminosidad alta.

En cuanto a la variable a, presentó una media de 0.56 unidades, valor que supera ligeramente el eje de los tonos verdes, desplazándose hacia los tonos rojos.
Para el caso de la variable b, la media fue de 9.78 unidades, ubicándose en la zona de los amarillos claros.

Tabla 1. Valores de $a^{*} / b^{*}, \Delta E^{*}, C^{*}$ y $H^{*}$ del sistema Hunter.

\begin{tabular}{ccccc}
\hline Muestra & $\boldsymbol{\Delta} \mathrm{E}^{*}$ & $\mathbf{H}^{*}$ & $\mathrm{C}^{*}$ & $\mathrm{a}^{*} / \mathrm{b}^{*}$ \\
\hline Promedio & 92,53 & 86,74 & 9,79 & 0,057 \\
M1 & 92,41 & 86,63 & 9,81 & 0,059 \\
M2 & 92,58 & 86,70 & 9,78 & 0,058 \\
M3 & 92,54 & 86,70 & 9,80 & 0,058 \\
M4 & 92,60 & 86,93 & 9,78 & 0,054 \\
\hline
\end{tabular}

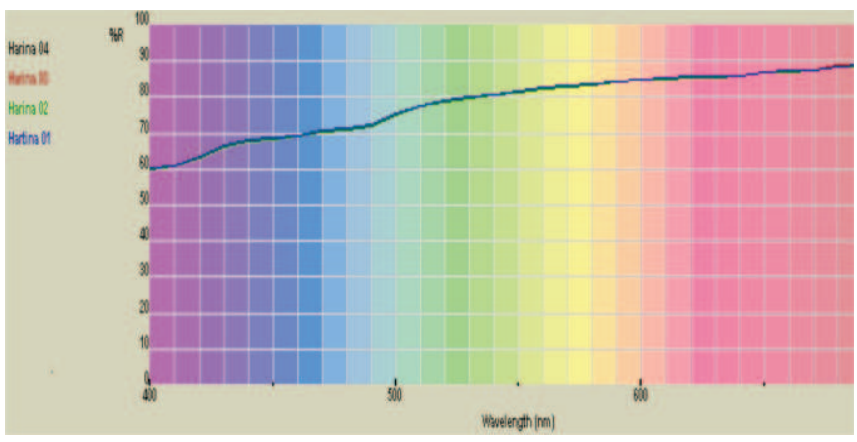

Figura 6. Correlación \% de reflexión Vs Longitud de onda

Las muestras presentaron uniformidad en cuanto a los tres factores obtenidos. Dado que en el color de la harina de trigo hay valores bajos para tonalidades verdes y amarillas, se calculó el cociente $\mathrm{a}^{*} / \mathrm{b}^{*}$, para obtener datos de referencia en el momento de comparar las muestras[13]. Este cociente tuvo un valor medio de 0.057 unidades, que representa el tono amarillo claro casi blanco típico de la harina de trigo.

\section{Índice de Blancura}

Esta es una medida de amplio uso en la industria textil y del papel y se ha extendido a la de medicamentos, plásticos, cerámica y alimentos.

Una superficie blanca ideal debe tener un índice de blancura (IW) igual a 100, cuando la diferencia entre el blanco ideal y el de la superficie que se evalúa se incrementa entonces IW disminuye.

El cálculo para determinar el indicador de blancura más indicado, de acuerdo a los datos obtenidos de factores $L^{*}$, a* $y b^{*}$ del sistema Hunter, son los siguientes:

1. $\mathrm{IW}=\mathrm{L}-3 \mathrm{~b}+3 \mathrm{a}$

Hunter (1960)

$\mathrm{IW}=92.014-3(9.78)+3(0.56)$

$\mathrm{IW}=64.35$ 
2. $\mathrm{IW}=100-((100 / \mathrm{L}) 2+(10 \mathrm{~b} 2)) 1 / 2$ Hunter $(1960)$

$\mathrm{IW}=100-((100 / 92.014) 2+(10 * 9.782)) 1 / 2$

IW $=69.06$

Para iluminante $C$. donde $L$, a y b son coordenadas cromáticas del sistema CIELab.

Los valores obtenidos por medio de las ecuaciones anteriores dieron valores muy similares de 64.35 y 69.06 , lo que indica que la harina de trigo no tiene un color blanco ideal, un valor muy similar al reportado por Colmenares $\mathrm{M}$ (et al), en la cual indica que las harina analizadas por ellos, presentan un color blanco amarillento.

\section{Actividad de Agua}

Los valores de la actividad de agua muestran la fracción del contenido de agua total de las muestras que está libre, y en consecuencia, disponible para el crecimiento de microorganismos y para que se puedan llevar a cabo diversas reacciones químicas que afectan a su estabilidad, el análisis de los datos efectivamente demostró que la muestra más vulnerable es la masa, obteniendo valores de 0.97 , como se indica en la Figura 10, lo cual fue altamente significativo ( $P$ valor $=0.00001)$.

\section{Means and 95,0 Percent Tukey HSD Intervals}

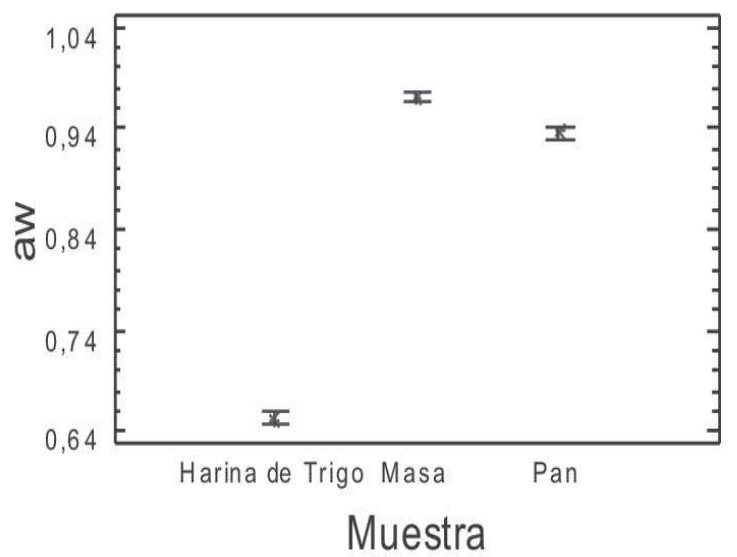

Figura 7. Valores de aw

Los valore de aw obtenidos están relacionados directamente con la textura de las muestras. Las muestras con una aw elevada como es el caso de la masa 0.97 y el pan 0.9357 tienen una textura más jugosa, tierna y masticable. En cambio, la harina con un valor de aw de 0.651 demuestra la textura propia de las harinas; sí su aw aumenta, la textura cambia, produciéndose el reblandecimiento de la harina (Pvalor $=0.00001)$.

\section{Humedad}

Means and 95,0 Percent Tukey HSD Intervals

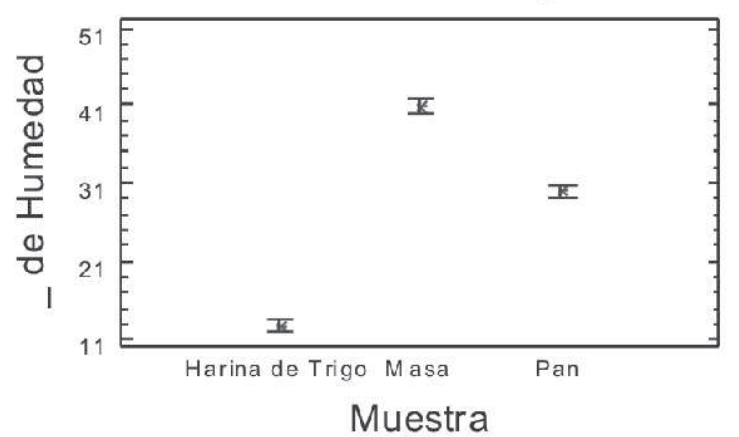

Figura 8. Valores de Humedad

El contenido de humedad es un factor de calidad en la presentación de productos de harinas y sus derivados, esta determinación permite evidenciar la evolución del producto desde la materia prima con valores de humedad promedio de $12.5918 \%$, valor similar a lo reportado por Wasiu et al. (2001), pasando por la elaboración de la masa en la cual el valor de la humedad alcanza valores de $40.8683 \%$, debido a la adición de agua lo que permite conseguir un producto pastoso y flexible; hasta llegar a la elaboración del pan con valores de humedad de $29.9504 \%$, humedad adecuada para este tipo de productos, (P valor $=0.00001)$.

Color

Means and 95,0 Percent Tukey HSD Intervals

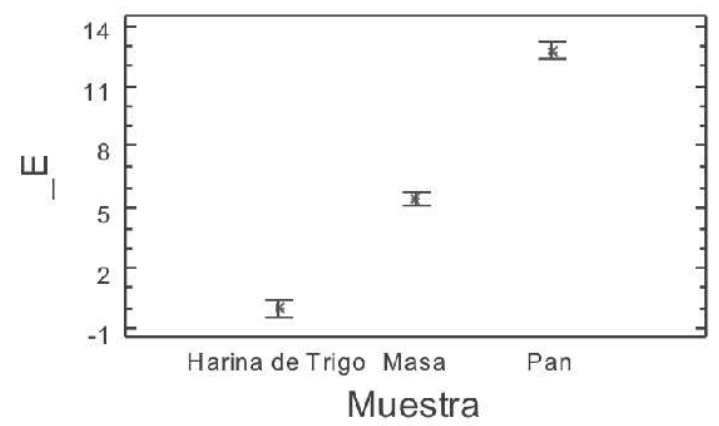

Figura 9. Determinación de color

El color ${ }^{[10]}$ es un atributo importante tanto para la harina como los productos elaborados a base de ella. De hecho, con frecuencia juzgamos la calidad de estos alimentos en función del color de los mismos. El color es la propiedad sensorial más importante asociada con el sentido de la vista aunque también existen otros atributos sensoriales detectados por medio de este sentido: apariencia, forma, superficie, tamaño y brillo. La primera impresión que se tiene de los alimentos generalmente es visual y su aceptación depende en mayor o menor medida de su color. La apreciación de esta cualidad puede dar lugar a ideas preconcebidas acerca de otros factores de calidad como son 
el sabor y el aroma.los resultados obtenidos muestran al cambio de color de la masa y el pan, respecto a la masa que en este caso hace de blanco, la gráfica demuestra como la masa a adquirido un valor de color significativa diferente al de la harina y en el caso del pan, el cambio es más representativo, ( $p$ valor $=0.00001$ )

\section{CONCLUSIONES}

Los análisis de calorimetría diferencial de barrido y el análisis térmico mecánico dinámico de los productos de panadería han demostrado que estos se obtienen desde materiales visco elásticos, los cuales después del tratamiento de cocción sus estructuras son generalmente consideradas para ser formadas por una continua red macromolecular basada sobre las proteínas del gluten y enlazadas por almidones cristalinos parcialmente granulares, las propiedades funcionales de las proteínas del gluten de trigo son principalmente la determinación de la estructura de la masa.

Durante el horneado modificaciones irreversibles del almidón y las proteínas a altas temperaturas y la deshidratación superficial son los responsables de la formación de la corteza del pan, en la parte central de la mezcla la gelatinización del almidón y la coagulación de la proteína inducen a la formación de la miga del pan, después del enfriamiento, el bajo contenido de agua en la corteza (3$7 \%$ ) es suficiente para la transición de los componentes del pan desde un estado gomoso a vítreo consecuentemente un endurecimiento estructural ocurre y es el responsable de la frescura de la corteza.

En la determinación del índice de blancura de acuerdo con los resultados obtenidos para las cuatro muestras, la harina de trigo comercial presenta tonos amarillos claros, con alta luminosidad.

Las muestras de harina presentaron tonos amarillo claro, presentando valores positivos de b comprendidos entre 9.77 y 9.79; es decir que tendieron al amarillo. Acusaron valores de a positivos, entre 0.52 y 0.58 por lo que se encontraron dentro de la zona de los tonos rojizos. Éstas muestras presentaron valores de a/b bajos, 0.057 .

El cociente $\mathrm{a} / \mathrm{b}$ tiene un valor de 0.057 indicando que las muestras son de color amarillo claro.

Los valores de $\Delta \mathrm{E}^{*}$ indican una representación tridimensional de los estímulos perceptuales del color. Como los puntos en el espacio (que representan los estímulos M1, M2, M3 y M4), son coincidentes, entonces la diferencia cromática entre ambos estímulos es igual a cero, indicando que no hay diferencia representativa entre las muestras.

Los valores de $\mathrm{C}^{*}$ y de $\mathrm{H}^{*}$ permiten interpretar que no hay diferencia cualitativa de color entre las muestras; así, en el eje del rojo-verde $a^{*}$, una muestra con mayor valor a* no se percibe necesariamente como "más rojo" lo que ocurre con la harina de trigo, que otra con menor valor a*. El uso de C*ab y $\mathrm{H}^{*}$ ab permite entonces una representación más intuitiva del color.

Los valores obtenidos para el índice de blancura, indican que la harina de trigo no tiene un color blanco ideal.

El colorímetro HunterLab resultó ser efectivo para determinar el color en la harina de trigo. Del análisis de los datos surgió que tanto el cociente a/b como los datos de, $\Delta \mathrm{E}^{*}, \mathrm{C}^{*}$ y $\mathrm{H}^{*}$ constituyen otro parámetro adecuado para comparar y clasificar harinas de trigo y brinda mayor información para la comercialización de este producto.

\section{BIBLIOGRAFÍA}

1. Decreto 1944 de octubre 28 de 1996

2. Daza, Carlos Hernán, M.D., M.Sc., M.P.H., "Mal nutrición de micronutrientes. Estrategias de prevención y control", http://colombiamedica.univalle.edu.co/index.html

3 Sunny Kim , 2005, OPS, “Código de prácticas para la fabricación de premezclas alimenticias”, Unidad de Nutrición, Área de Salud Familiar y Comunitaria, Washington D.C.

4. Harrell-Bond BE, Henry CJK, Wilson K. Fortification of Foods for Refugees. Lancet 1989; 1392 SCN News 4, 29-30.

5. King J, De Pablo S. Pérdidas de vitaminas durante el procesamiento de los alimentos (Vitamins losses in food processing). Rev Chil Nutr 1987; 15(3):143-152

6. J.C. Morales González; A. Mantilla Quijano: El derecho a la alimentación en Colombia: Situación, contextos y vacios. Una aproximación al compromiso del Estado colombiano a la luz de las Directrices Voluntarias sobre el Derecho a la Alimentación. Bogotá; diciembre de 2007

7. Informe sobre el Desarrollo Mundial 2000/2001: Lucha contra la Pobreza" Banco Mundial Paginas: Completo

8. P. E. A. Andang'o, S. J. M. Osendarp, R. Ayah, C. E. West, D. L. Mwaniki, C. A. De Wolf, R. Kraaijenhagen, F. J. Kok and H. Verhoef, The Lancet, 369, 1799-1806

9. Sanny S.L. Chan, Elaine L. Ferguson, Karl Bailey, Umi Fahmida, The concentrations of iron, calcium, zinc and phytate in cereals and legumes habitually consumed by infants living in East Lombok, Indonesia, Journal of Food Composition and Analysis 20 (2007) 609-617

10. Tester, R.f., Debon, S.j. Annealing of starch: a review. En: International journal of biological macromolecules. Vol. 27 (2000); p. 1-12.

11. Biliaderis, C.G. Structures and phase transitions of starch in food systems. En: Food Technology. (1992); p. 98-109,145

12. Wang, L. Z.; White, P. J. Structure and properties of amylose, amylopectin and Intermediate materials of oat starches. Cereal Chemistry, v. 71, n. 5, p. 263-268, 1994b.

13. Billmeyer, F.W. y Saltzman, M (1981) Principles of color technology. John Wiley \& sons. New York. 